\title{
TENDENCIAS EN EL CONSUMO DE ALIMENTOS EN LAS SOCIEDADES PREHISPÁNICAS DE QUEBRADA DE HUMAHUACA (JUJUY, ARGENTINA)
}

\author{
TRENDS IN FOOD CONSUMPTION IN THE PRE-HISPANIC SOCIETIES OF \\ QUEBRADA DE HUMAHUACA (JUJUY, ARGENTINA
}

\author{
Violeta A. Killian Galván ${ }^{1,2}$, Verónica Seldes ${ }^{1,3}$, Clarisa Otero ${ }^{1,2,4}$, M. Clara Rivolta ${ }^{2,5}$ y Axel E. Nielsen ${ }^{1,6}$
}

\begin{abstract}
El objetivo de este trabajo es presentar la composición isotópica del carbono $\left(\delta^{13} \mathrm{C}\right.$ y $\delta^{13} \mathrm{C}$ ) y del nitrógeno $\left(\delta^{15} \mathrm{~N}\right)$ del registro óseo humano de 19 individuos hallados en los sitios arqueológicos de Quebrada de Humahuaca (Jujuy, Argentina), asociados a las ocupaciones comprendidas entre el Formativo Tardío (ca. 500-900 AD), el periodo de Desarrollos Regionales (900 - 1430 AD) y el periodo Incaico (ca. 1430 AD - 1536 AD). A su vez, se consideraron resultados ya publicados, llegando a 41 individuos analizados para el área de estudio. Se discuten los aportes de la reconstrucción paleodietaria en la caracterización de estas sociedades teniendo en cuenta los modelos de organización social y productiva propuestos para la región. La aproximación utilizada para el análisis de datos fue la construcción de un modelo de mezcla bayesiano, integrando los valores isotópicos de vegetales actuales de Quebrada de Humahuaca y especímenes de fauna camelidae provenientes de las provincias fitogeográficas de puna y prepuna de Jujuy. Nuestros resultados indican que las proporciones de los recursos de la dieta no habrían variado considerablemente a lo largo del tiempo, siendo el maíz (y/o el amaranto) el recurso predominante desde los tiempos de la consolidación de las economías agropastoriles.
\end{abstract}

Palabras claves: paleodieta, isotopos estables, cambio social, bioarqueología.

The aim of this paper is to present the carbon $\left(\delta^{13} C_{\text {collagen }}\right.$ and $\left.\delta^{13} C_{\text {apatite }}\right)$ and nitrogen $\left(\delta^{15} N\right)$ isotopic compositions of the human bone record of 19 individuals found in archaeological sites of Quebrada de Humahuaca (Jujuy, Argentina), associated with occupations between the Late Formative (ca. 500-900 AD), the Regional Development Period (900 - 1430 AD) and the Inca Period (ca. 1430 AD - 1536 AD). Previously published, results were also considered, allowing for a total of 41 individuals analyzed for the study area. The characterization of these societies by paleodietary reconstruction is discussed considering models of social and productive organization proposed for the region. A Bayesian mixing model was used for data analysis, integrating isotopic values of modern plants from Quebrada de Humahuaca and camelidae fauna specimens from the phytogeographic provinces of Puna and Prepuna of Jujuy. Our results indicate that diet composition did not vary considerably over time, with maize (and/or amaranth) being the predominant resource since the time of consolidation of the agro-pastoral economies.

Key words: Paleodiet, stable isotopes, social change, bioarchaeology.

Los patrones alimentarios de una sociedad, esto es, los hábitos o prácticas alimentarias de un grupo, son el resultado de la interacción de múltiples variables, biológicas, ecológicas, tecnológicas, económicas, sociales, políticas e ideológicas, siendo las características intrínsecas y/o nutritivas de los alimentos un aspecto más entre otros a la hora de definir su relevancia en la dieta cotidiana. De hecho, se postula que la comida y su modo de elaborarla constituyen una de las prácticas que otorgan identidad a los pueblos (Bray 2003; Contreras Hernández 1993). Ejemplo de ello es el consumo del maíz (Zea mays) en la extensa área andina. Las crónicas hispano-indígenas colocan a este cultígeno como un recurso central en el repertorio culinario, al menos durante el incanato, siendo indispensable

\footnotetext{
${ }^{1}$ Consejo Nacional de Investigaciones Científicas y Técnicas (CONICET), Buenos Aires, Argentina.

${ }^{2}$ Instituto Interdisciplinario Tilcara, Facultad de Filosofía y Letras, Universidad de Buenos Aires, Buenos Aires, Argentina. violetakillian@gmail.com; rivoltaclara@gmail.com

${ }^{3}$ Instituto de Ciencias Antropológicas, sección Antropología Biológica, Facultad de Filosofía y Letras. Universidad de Buenos Aires, Buenos Aires, Argentina. vseldes@gmail.com

${ }^{4}$ Instituto de Datación y Arqueometría (InDyA) Jujuy, Argentina. clarisaotero@gmail.com

${ }^{5}$ Facultad de Humanidades, Universidad Nacional de Salta, Salta, Argentina.

${ }^{6}$ Instituto Nacional de Antropología y Pensamiento Latinoamericano (INAPL), Buenos Aires, Argentina. axelnielsen@gmail.com
} 
en todas las instancias celebrativas (Métraux 1972). La arqueología, a su vez, expuso su relevancia en las sociedades prehispánicas siendo su producción y consumo una de las problemáticas más estudiadas, buscando establecer su rol y el ritmo de su integración en las economías (Alfonso-Durruty et al. 2017; Hastorf 1985; Ikehara et al. 2013; Lantos et al. 2015; Oliszewski 2012; Staller 2006; entre otros). Estos estudios mostraron la importancia de las decisiones políticas, económicas y simbólicas, antes que el mero condicionamiento ambiental para definir su lugar en las estrategias agrícolas (Burger y van der Merwe 1990; Hastorf 2003; Killian Galván 2016a; Santana-Sagredo et al. 2016; Somerville et al. 2015; Staller 2006; entre otros).

En el Noroeste Argentino (NOA), región meridional de los Andes, la relevancia del maíz en la dieta de las poblaciones prehispánicas ha sido explorada mediante diferentes líneas de evidencia. Aunque se han presentado matices (Haber 2006; Ortiz 2007; Quesada 2007), se ha mantenido casi incuestionada la existencia de una tendencia progresiva a una mayor intensificación de la producción agropastoril (Nielsen 1997; Olivera 1992; Tarragó 2000). Asimismo, se ha propuesto la existencia de una tendencia, desde la transición al agropastoralismo hasta la expansión incaica, hacia el equilibrio entre el consumo cárnico y vegetal, en particular a partir de la incorporación de maíz a la dieta (Olivera y Yacobaccio 1999). No obstante, múltiples análisis de microfósiles sobre terrazas de cultivo en diferentes sitios arqueológicos tardíos e incaicos de las áreas valliserranas y quebradeñas del NOA han puesto de manifiesto la escasa representatividad del cultivo de maíz (Korstanje 2015). A su vez, a partir del análisis de isótopos estables sobre restos humanos, se propuso que si bien el maíz fue relevante en gran parte de las economías de la macrorregión, no tuvo una tendencia hacia un mayor consumo efectivo como resultado de los cambios demográficos y la intensificación productiva postulados para el área (Killian Galván 2018). Estos estudios mostraron, al menos de manera exploratoria, cierta coherencia con lo hallado en las crónicas hispano-indígenas de Perú (de Molina 1959 [1575]), donde se relata que las sociedades que ocuparon los pisos altitudinales más elevados, como podría ser el caso de las sociedades puneñas del NOA, cubrieron su alimentación con alimentos cuyo crecimiento es más exitoso en condiciones de aridez y bajas temperaturas, como es el caso de la quínoa (Chenopoduium quinoa) y los tubérculos. No obstante, se propuso que el maíz pudo ser un elemento utilizado en instancias celebrativas, compartido por la comunidad en momentos específicos, pero su producción y el énfasis en su intercambio pudo no estar acompañado por el hábito de su consumo diario, sino que pudo ser un alimento lujoso (sensu Hastorf 2003), compartido por la comunidad en ocasiones especiales (Killian Galván et al. 2016a).

Siguiendo esta línea de trabajo, discutiremos aquí el lugar del maíz en los patrones de consumo de las sociedades prehispánicas en una de las áreas más estudiadas por la arqueología en el NOA, la Quebrada de Humahuaca. Esta quebrada, localizada en la actual provincia de Jujuy, cuenta con una ocupación casi sin interrupciones de 10 mil años (Fernández Distel 1980). Con tal fin, se realizaron análisis de isótopos estables de carbono en la fracción orgánica $\left(\delta^{13} \mathrm{C}_{\mathrm{col}}\right)$ e inorgánica $\left(\delta^{13} \mathrm{C}_{\mathrm{ap}}\right)$ y del nitrógeno $\left(\delta^{15} \mathrm{~N}\right)$ de los restos óseos de 19 individuos humanos hallados en sitios arqueológicos adscritos a las ocupaciones comprendidas entre el periodo Formativo Tardío (ca. 500-900 AD), de Desarrollos Regionales (900-1430 AD) e incaico (ca. $1430 \mathrm{AD}$ - $1536 \mathrm{AD}$ ), a los que se sumaron valores isotópicos procedentes de otras investigaciones en el área (Amman et al. 2014; Gheggi et al. 2018; Killian Galván 2018; Killian Galván et al. 2016b; Lynch Ianniello et al. 2018). Esta vía analítica resulta adecuada, dado que nos permite conocer, por un lado, el patrón fotosintético dominante en la dieta asimilada y, por otro, el lugar dentro de la cadena trófica que ocuparon los individuos bajo análisis. Por lo tanto, resulta una línea de evidencia independiente, sensible a los cambios que pudieron suscitarse a nivel individual en la alimentación de poblaciones del pasado. Se utilizó el modelo de mezcla bayesiano FRUITS (Food reconstruction Using Isotopic transferred Signals) diseñado por Fernandes et al. (2014), el cual permite utilizar de manera simultánea diferentes proxies paleodietarios a la vez que requiere la inclusión de datos isotópicos sobre las posibles fuentes de recursos alimenticios locales.

\section{Cambios Económicos en la Quebrada de Humahuaca Prehispánica}

En la arqueología del NOA, el pasaje de las sociedades cazadoras-recolectoras a las productivas se englobó bajo el término Formativo, implicando cambios económicos como la consolidación del pastoreo y en menor escala de la agricultura, en sociedades ya sedentarias y en el marco de una vida aldeana (Olivera 1992). Si bien existieron intentos por quitarle una connotación temporal al concepto, prevaleció la visión de asignarle una cronología específica, como es el intervalo entre los 3500 y 1500 años AP (Olivera y Grant 
2009). No obstante, se ha planteado que recién hacia los 2500 años AP aparecerían este tipo de economías de manera más extendida en el NOA (Raffino 1993; Muñoz y Stenborg 1999; Olivera y Palma 1986; Palma y Olivera 1992-1993). Las evidencias de las primeras economías productoras de alimentos, con fechados previos a los 3000 años AP en el área de Quebrada de Humahuaca son escasas, contándose con los sitios Inca Cueva y Huachichocana III, puestos de actividades no permanentes, probablemente vinculados a otros sitios de ocupación más prolongada (Olivera y Palma 1997). Es en Huachichocana donde se encuentran evidencias de consumo de especies vegetales que presentan algún grado de manejo humano, como es el caso del ají (Capsicum aff. chacoense) (Lema 2014). Posteriormente, hacia los comienzos del primer milenio de la era cristiana, aparecen sitios residenciales como Antumpa, Estancia Grande y el Alfarcito, ubicados en áreas ecotonales de quebradas subsidiarias a la Quebrada de Humahuaca, un entorno donde, a diferencia del valle principal, fenómenos de erosión y depositación más moderados han permitido la preservación de los sitios tempranos. Serían sistemas de explotación mixtos, hallándose evidencias de pastoreo y caza de camélidos, así como de horticultura, con canchones emplazados en conos de deyección en zonas de considerable altura (Olivera y Palma 1997). Particularmente, el desarrollo agrícola en Alfarcito fue vinculado en sus ocupaciones tempranas (ca. 2000 años AP) con los sitios de tradición San Francisco, ubicados en las yungas jujeñas, donde si bien aún son escasas las evidencias de especies vegetales domesticadas, se ha propuesto el probable consumo de maíz en el marco de una horticultura de baja escala (Ortiz et al. 2014). Por su parte, casos como el de Antumpa en la cabecera de la Quebrada, ofrece condiciones más propicias para los cultivos microtérmicos (Leoni et al. 2012). Con cronologías posteriores se encuentran los sitios Til 20 y Til 22, rescates arqueológicos en la actual ciudad de Tilcara, sobre la Quebrada troncal (Mendonça et al. 1991; Rivolta 1996, 2005), los cuales también brindaron elementos para caracterizar a las ocupaciones permanentes del primer milenio. Se trataría de aldeas estables o semiestables emplazadas en lugares con acceso directo a cursos de agua permanente o en las desembocaduras de cauces estacionales (Leoni et al. 2012; Nielsen 2001; Olivera y Palma 1986). Las aldeas habrían sido habitadas por pequeños grupos que obtenían los recursos básicos para su subsistencia en el entorno cercano, sin necesidad de gran inversión tecnológica para la práctica del riego (Nielsen 1996).

Con el tiempo, hacia ca. $900 \mathrm{AD}$, se inicia lo que la arqueología denominó período de Desarrollos
Regionales, el cual puede segmentarse a su vez en subperiodos: PDRI (900-1250 DC) y PDRII (12501430 DC). En la Quebrada de Humahuaca este periodo se caracterizó por fenómenos de redistribución poblacional e integración política y económica que culminaron con la formación de grandes unidades territoriales. Para autores como Tarragó (2000) y Raffino (2007) se iniciaría en este periodo la explotación agrícola a gran escala, como también la segregación entre los loci residenciales y agrícolas (Raffino 2007). En lo que refiere al pastoreo de camélidos, es probable que a partir de este momento se practicara el traslado estacional de rebaños entre las quebradas altas. De este modo, se aprovecharían los pastos tiernos de verano y zonas más bajas durante la estación seca, el invierno, donde los animales se alimentarían de forrajes no estacionales y de los desechos de cultivo disponibles en los rastrojos luego de la cosecha. Tal situación pudo ser particularmente relevante en el sector medio de la Quebrada de Humahuaca, pues, como indican Mercolli et al. (2014), la cantidad y calidad de forrajes suelen ser mayores hacia el sector norte y sur de la misma. A su vez, Olivera y Yacobaccio (1999) también consideran que habría una intensificación en las redes de intercambio garantizada por la complementariedad ecológica. Este intercambio a gran escala haría esperar una dieta variada en recursos, gracias al acceso a bienes alóctonos.

Durante el periodo de Desarrollos Regionales II (PDRII), el proceso de concentración de la población se habría acelerado, culminando con la formación de asentamientos conglomerados de alta visibilidad y difícil acceso, distribuidos a lo largo del Río Grande y porciones inferiores de las quebradas laterales, como Hornillos, Juella y Los Amarillos. El abandono del uso residencial de zonas estratégicas para la producción permitió ganar protección y seguridad en un escenario de alta conflictividad social (Nielsen 1996). Las formaciones sociales resultantes mostrarían diferencias estructurales organizadas en base a principios corporativos y de integración segmentaria (Nielsen 2006a). Este escenario se habría visto acompañado por una intensificación de las explotaciones, evidenciada en los inicios de la construcción de sitios agrícolas a considerable distancia de las bases residenciales (p.ej., los sitios arqueológicos El Alfarcito y Coctaca), la consolidación de la trashumancia pastoril testimoniada por ocupaciones temporarias vinculadas a esta actividad en quebradas altas (p.ej., los sitios Tomayoc-Lavallée et al. 1997- y Huachichocana III -Madero 1993) y la disminución de la caza (Madero 1993). Asimismo, una tendencia clara a partir del Formativo Tardío e intensificada en el periodo de Desarrollos Regionales es la 
mayor especialización en los morfotipos de los rebaños, buscándose aquellos útiles para la lana y el transporte (Mercolli et al. 2014). Es probable que estos cambios hayan sido acompañados por cierta diferenciación ocupacional fruto de la integración de la población bajo estrategias económicas comunitarias (Nielsen 2001, 2006b).

Por su parte, durante el periodo Incaico (ca. 1430 - 1532 AD), con el avance y anexión de territorios por parte del Imperio, existió un afianzamiento de la agricultura intensiva (con cuadros, terrazas y obras de riego), desarrollándose verdaderos centros regionales de producción para el Estado. En Quebrada de Humahuaca el principal de ellos se ubica en el complejo Coctaca-Rodero, hacia el norte. En el Pucará de Tilcara, en cambio, se habría establecido un centro de producción de bienes suntuarios confeccionados en piedras semipreciosas (alabastro, ónix) y metales, como lo evidencia la presencia de numerosos talleres dedicados a estas labores (Greco y Otero 2016; Krapovickas 1983; Nielsen 2001; Otero y Tarragó 2017).

A lo largo de este extenso lapso temporal sería esperable que los cambios estructurales en las estrategias agropastoriles influenciaran la vida cotidiana de las comunidades quebradeñas. Por lo tanto, entendemos que la segmentación propuesta (periodos Formativo, PDRI, PDRII e Inca) no obedece a simples recortes temporales puramente arbitrarios, sino que resultan de discontinuidades históricas objetivas en las sociedades bajo análisis y que resultan útiles a la hora de evaluar el devenir de los patrones de consumo de alimentos.

\section{Área de Estudio y Sitios Arqueológicos Discutidos}

Ubicada en el sector central de la provincia de Jujuy, la Quebrada de Humahuaca (23 $10^{\prime}$ y $23^{\circ} 50^{\prime} \mathrm{S} ; 65^{\circ} 20^{\prime} \mathrm{O}$; Figura 1 ) constituye un estrecho valle árido surcado por el Río Grande de Humahuaca; tiene un recorrido $\mathrm{N}$ - S de casi $100 \mathrm{~km}$ y se encuentra limitada hacia el este por los cordones montañosos de Zenta - Aparzo Hornocal - Tilcara y hacia el oeste por el de Aguilar - Mal Paso - Chañi, recibiendo el aporte de varias quebradas laterales que segmentan dichas serranías (Albeck 1992; Cabrera 1976). Comprende altitudes desde los 2.000 hasta los $3.700 \mathrm{msm}$ en el fondo de valle, mientras que las cumbres de las serranías que la enmarcan superan los $5.000 \mathrm{msm}$. La región pertenece, fitogeográficamente, a la Provincia Prepuneña, lindando hacia el oeste con la Puna y hacia el este con las selvas de Yungas. Se caracteriza por la presencia dominante de estepas arbustivas, y churqui (Prosopis ferox) y cactáceas (Trichocereus pasacana). También se encuentran algarrobo (Prosopis alba, Prosopis nigra), y por encima de los $3.700 \mathrm{msm}$, bosquecillos de queñoa (Polylepis tomentella) (Cabrera 1976). Posee características geográficas que han favorecido la producción agrícola, principalmente gracias a su gradiente altitudinal, que permite la diversidad productiva y la alta heliofanía. Este factor, sumado a la escasa humedad (con precipitaciones anuales de $136 \mathrm{~mm}$ y temperatura media anual de $12^{\circ} \mathrm{C}$ ) y la altura (ca. de $2.500 \mathrm{msm}$ ), permiten el cultivo de productos agrícolas con óptimas propiedades organolépticas, frutas y hortalizas con una alta resistencia al estrés (Rodríguez 2009).

Tomando en consideración las posibilidades de desarrollo productivo, el área puede ser dividida en tres zonas diferentes: (1) por debajo de los 3.100 msm formada por los fondos de valle, incluyendo los del Río Grande y cursos tributarios, zona apta para el cultivo de especies mesotérmicas como el maíz (Zea mays), además del poroto (Phaseolus vulgaris), ají (Capsicum anuum) y calabaza (Curcubita pepo) en sectores más reparados; (2) entre $\operatorname{los} 3.100$ y $3.500 \mathrm{msm}$ zona ideal para la producción de productos microtérmicos como papa (Solanum tuberosum), oca (Oxalis tuberosa), quinoa (Chenopodium quinoa Willdenow) y kiwicha (Amaranthus caudatus), además de contar con buena oferta de pasturas y; (3) las laderas de las montañas donde prosperan recursos aptos para el pastoreo (llama, Lama glama) y la caza, mayormente guanaco (Lama guanicoe), vicuña (Vicugna vicugna), y en las serranías hacia el oriente, taruca (Hippocamelus antisensis), y en menor medida quirquincho (Chaecttabla ophractus nationis) y vizcacha (Lagidium lockwodi) (Nielsen 2001). Vale notar que todos los sitios estudiados se encuentran en la primera zona mencionada, especialmente apta para la agricultura de maíz y otras especies mesotérmicas.

\section{Descripción de los sitios arqueológicos}

Flores 1: Se emplaza en el sector urbano de la localidad de Tilcara $(2.450 \mathrm{msm})$. Fue asignado al periodo Formativo Tardío por la similitud de los materiales hallados utilizados como indicadores cronológicos y su proximidad con el sitio "Til 20", paradigmático de dicho período (Rivolta 1996; Tarragó y Albeck 1997). Los restos óseos humanos han sido recuperados en un trabajo de arqueología de rescate (Zaburlin et al. 2006), identificándose eventos de estrés aislados y un importante desgaste dental en la mayoría de los individuos analizados (Seldes 2007). 


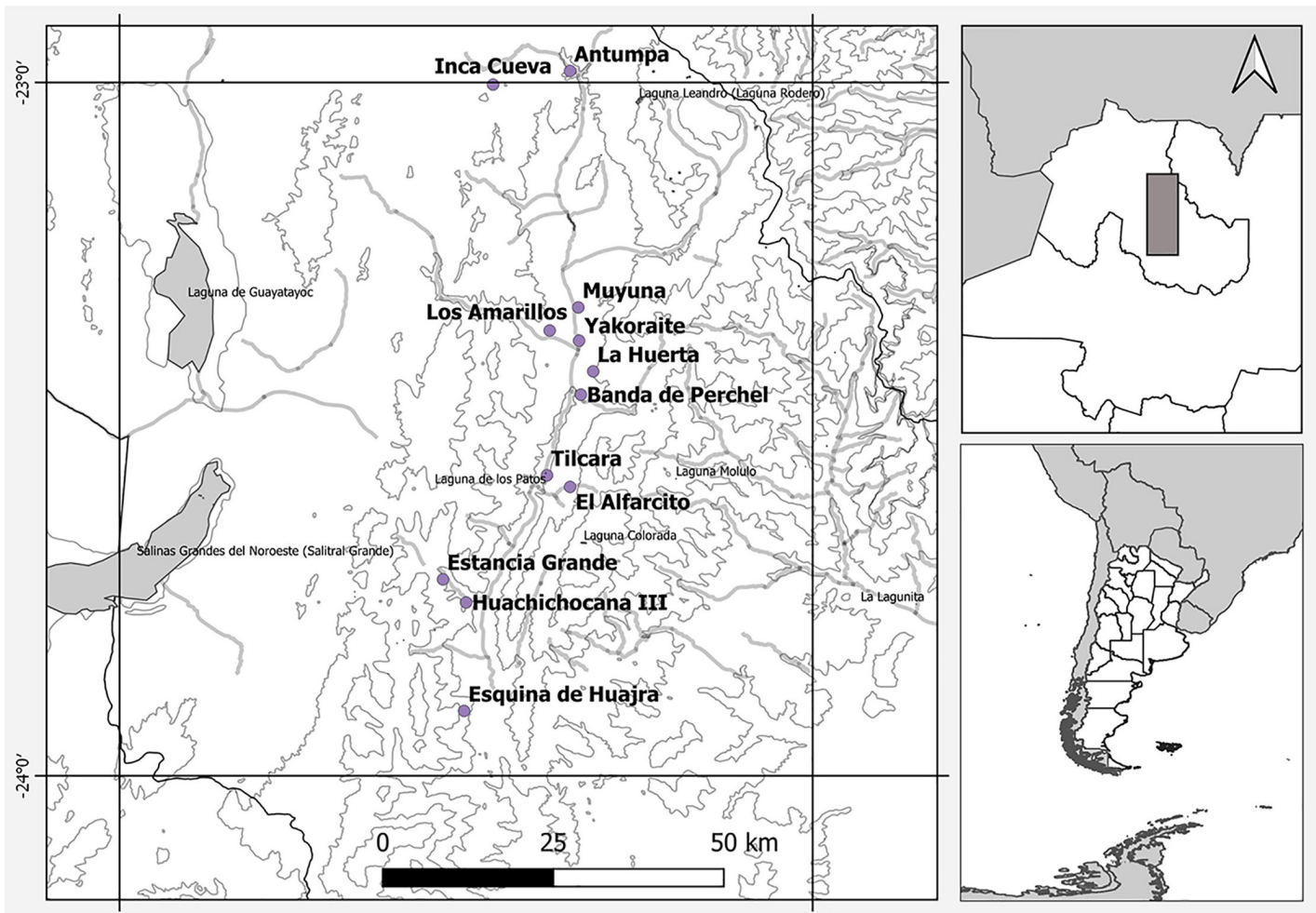

Figura 1. Sitios arqueológicos de la Quebrada de Humahuaca mencionados en este trabajo. Datos para la elaboración del mapa extraídos de www.openstreetmap.org (@ Colaboradores de OpenStreetMap) y del Instituto Geográfico Nacional de la República Argentina.

Archaeological sites of Quebrada de Humahuaca mentioned in this work. Data for the preparation of the map obtained from www.openstreetmap.org (@) OpenStreetMap collaborators) and Instituto Geográfico Nacional de la República Argentina.

Muyuna: se trata de un asentamiento residencial, correspondiente al PDRI, que se encuentra ubicado sobre una terraza fluvial alta en la margen izquierda del Río Grande y a una altitud de $2.850 \mathrm{msm}$. El material cerámico recuperado en las excavaciones corresponde a los estilos Alfarcito Polícromo e Isla Polícromo, propios del periodo en cuestión (Nielsen 2007), y cuenta con un fechado radiocarbónico que le da una profundidad temporal de 1.000 años (Nielsen 1997b). El análisis bioarqueológico efectuado sobre la serie esqueletal permitió inferir una baja exposición a factores de estrés de tipo nutricional (Seldes 2007).

Banda de Perchel: también con una cronología correspondiente al PDRI, se trata de un sitio ubicado a $2 \mathrm{~km}$ al sur de la localidad de Huacalera, sobre la margen izquierda del Río Grande de Jujuy y emplazado a $2.600 \mathrm{msm}$. El sitio cuenta con restos alfareros correspondientes al estilo Isla. Asimismo, en un perfil localizado en proximidad a la senda vecinal, se relevaron dos tumbas, de las que se recuperaron restos óseos humanos asociados a puntas de proyectil y una vincha de cobre en mal estado de conservación (Rivolta 2005).

Pucará de Tilcara (Til 1): Emplazado a 2.460 $\mathrm{msm}$, se trata del poblado prehispánico de mayor extensión de la Quebrada, ocupado desde ca. 1100 AD hasta la ocupación europea. Durante la dominación incaica funcionó como cabecera política de la región (Otero 2013). Se analizaron tres individuos correspondientes al periodo Incaico, hallados durante un rescate arqueológico. Este último contexto mortuorio presentó tres puntas de proyectil y cerámica Humahuaca-Inca.

\section{Materiales y Métodos Analíticos}

La muestra analizada se compone de 19 individuos humanos adultos, cuatro de los cuales corresponden al periodo Formativo Tardío, 12 al periodo de Desarrollos Regionales I y tres al periodo Incaico. En la mayoría de los casos las condiciones de preservación y el estado de completitud de las muestras impidieron la determinación de sexo y mayor precisión en la 
edad al momento de la muerte. El pretratamiento de las muestras óseas para el análisis isotópico, tanto en la fracción orgánica como inorgánica, se realizó en el Laboratorio de Biogeoquímica del Instituto de Geocronología y Geología Isotópica (INGEISCONICET/UBA). Para la extracción de colágeno se siguió el protocolo de Tykot (2004). Se procedió a la desmineralización del hueso mediante el uso de $2 \%$ de ácido clorhídrico durante $72 \mathrm{~h}$. Los contaminantes solubles fueron removidos usando $0.1 \mathrm{M}$ de hidróxido de sodio (24 h antes y después de la desmineralización). Respecto a la extracción de hidroxiapatita, se sometió a las muestras a hipoclorito de sodio al $2 \%$ durante $72 \mathrm{~h}$ para la eliminación de la materia orgánica (Tykot 2004). A este procedimiento le continúa la eliminación de carbonatos posdepositacionales, mediante el uso de un buffer de ácido acético al 0,1 M durante cuatro h (Garvie-Lok et al. 2004). El hueso, tanto para la fracción mineral como el colágeno, fue pesado antes y después de someterse a estos procedimientos, con el fin de evaluar el rendimiento obtenido. Las mediciones de los valores $\delta^{13} \mathrm{C}$ y $\delta^{15} \mathrm{~N}$ fueron realizadas en un analizador elemental Carlo Erba Elemental Analyser (CHONS), acoplado a un espectrómetro de masas de relaciones isotópicas de flujo continuo Finnigan MAT Delta V (CFIRMS), a través de una interface Thermo ConFlo IV y utilizando estándares internos. El análisis de la composición isotópica de carbonatos fue realizado utilizando el método de ácido fosfórico desarrollado por McCrea (1950), incluyendo modificaciones posteriores (Panarello et al. 1980). El espectrómetro utilizado para las muestras de hidroxiapatita fue un Delta S Finnigan Mat triple colector.

\section{Reconstrucción cuantitativa de la dieta}

Para la reconstrucción cuantitativa paleodietaria optamos por el modelo de mezcla bayesiano FRUITS (Food Reconstruction Using Isotopic Transferred Signals), modelo que ha mostrado ser eficiente a la hora de estimar la probabilidad de las contribuciones de diferentes fuentes, sobre todo cuando los diferentes macronutrientes de los alimentos contribuyen en proporción variable a la señal isotópica de los tejidos del consumidor (Fernandes 2016). El modelo nos permite manejar diferentes incertidumbres, tales como aquellas asociadas a las señales isotópicas de los grupos de alimentos potenciales, los fraccionamientos isotópicos entre la dieta y los tejidos y la contribución relativa de los macronutrientes (Fernandes 2016; Fernandes et al. 2014). Asimismo, este modelo permite introducir información preexistente, como es el caso de aquella procedente de estudios fisiológicos o metabólicos (Fernandes et al. 2014).

Para la reconstrucción de la ecología isotópica y la conformación de grupos de recursos alimenticios se utilizó información de vegetales de consumo humano procedentes del sector sur y medio de Quebrada de Humahuaca (publicados en Gheggi y Williams 2013 y Killian Galván et al. 2015, respectivamente). En lo que concierne a los valores isotópicos en fauna, fue considerada la familia Camelidae, aquella mayormente representada en los sitios arqueológicos del área (Belotti López de Medina et al. 2016), recurriendo a los valores isotópicos aportados por las investigaciones de Mengoni Goñalons (2007). No obstante, dado que las investigaciones del primer autor corresponden al sur del área (sitios arqueológicos Esquina de Huajra y Volcán) y, por lo tanto, con características ecológicas poco representativas para el resto de Quebrada de Humahuaca, consideramos adecuado incluir la información isotópica procedente de las investigaciones de Samec et al. (2018) y de Killian Galván et al. (2016a) sobre camélidos actuales y arqueológicos, procedentes de las cuencas de la Puna de Jujuy más próximas a nuestra área de investigación. En la Tabla 1 se sintetiza la estadística descriptiva de los conjuntos de recursos ingresados en el modelo. Dentro del conjunto de recursos "Plantas C 4 /CAM" se agruparon especímenes de Zea mays, Amaranthus caudatus y Opuntia sp. En el correspondiente a "Plantas $\mathrm{C}_{3}$ " se agruparon Solanum tuberosum, Oxalis tuberosa, Chenopodium quinoa, Cucurbita ficifolia y Cucurbita sp. Dentro del conjunto "Camelidae", se han incluido Lama glama, Vicugna vicugna, Lama sp. (uno de los especímenes probablemente sea L. guanicoe).

El contenido nutricional de los grupos de alimentos (proteínas, carbohidratos y lípidos) fue expresado como contenido de carbono en peso seco, con los carbohidratos y lípidos combinados en una misma fracción. Para la estimación de la composición a nivel de macronutrientes de los alimentos se utilizó la información suplementaria sintetizada en el archivo 2, Tabla 1 de la publicación de Fernandes (2016).

Los valores isotópicos de los animales terrestres fueron estimados a partir de los valores en colágeno óseo, considerando el fraccionamiento isotópico entre los valores isotópicos de macronutrientes y colágeno para este tipo de organismos propuesto por Fernandes (2016) y basado en una síntesis de las publicaciones más relevantes abocadas a discutir las discriminaciones isotópicas. Los fraccionamientos para los animales terrestres considerados fueron: $\delta^{13} \mathrm{C}_{\text {protéína-colágeno }}-2 \% o, \delta^{13} \mathrm{C}_{\text {lípidos-colágeno }}-8 \% o, \delta^{15} \mathrm{~N}_{\text {proténa- }}$
colágeno
$+2 \% o$, con una incertidumbre del $1 \%$. Debido 
Tabla 1. Valores isotópicos de los grupos de recursos utilizados en el modelo de mezcla bayesiano.

Isotopic values of the resource groups used in the Bayesian mixing model.

\begin{tabular}{ccccccc}
\hline \multirow{2}{*}{ Recursos } & \multicolumn{2}{c}{$\delta^{13} \mathrm{C}(\% \circ)$} & \multicolumn{3}{c}{$\delta^{15} \mathrm{~N}(\% \circ)$} \\
\cline { 2 - 7 } & $\mathrm{n}$ & Media & $\mathrm{DS}$ & $\mathrm{n}$ & Media & $\mathrm{DS}$ \\
\hline Plantas $\mathrm{C}_{4} / \mathrm{CAM}$ & 37 & $-10,6$ & 3,6 & 36 & 5,1 & 2,2 \\
\hline Plantas $\mathrm{C}_{3}$ & 13 & $-24,9$ & 0,9 & 13 & 6,9 & 1,5 \\
\hline Camelidae & 21 & $-15,6$ & 2,4 & 21 & 8,0 & 2,4 \\
\hline
\end{tabular}

a que los valores $\delta^{13} \mathrm{C}_{\text {ap }}$ son un proxy paleodietario que implica el carbono producto de una mezcla de fuentes, la señal $\delta^{13} \mathrm{C}$ de cada grupo de alimentos fue aquella del carbono total (bulk). Por otro lado, los valores $\delta^{13} \mathrm{C}$ totales de los animales terrestres fueron estimados como una media ponderada (en relación con la composición nutricional) de los valores $\delta^{13} \mathrm{C}$ de lípidos y proteínas.

Los valores isotópicos de las plantas modernas fueron ajustados considerando el efecto Suess (+1,5\% ; Craig 1957). A su vez, se tuvo en cuenta el fraccionamiento existente entre los valores isotópicos $\delta^{13} \mathrm{C}$ del total de las plantas y los de las proteínas de estas $(-2 \%$ ) y entre dicho total y los carbohidratos (ca. +0,5\%) (Fernandes 2016; Tieszen 1991).

En lo que respecta a la discriminación isotópica entre la dieta y los tejidos, Fernandes (2016) sugiere considerar tanto los trabajos experimentales sobre mamíferos omnívoros y aquellos en humanos en tejidos de recambio rápido (p.ej., sangre, pelo). Entonces, los fraccionamientos isotópicos para los valores $\delta^{13} \mathrm{C}$ entre la dieta y el colágeno óseo $\mathrm{y}$ entre la dieta y la bioapatita humanos se tomaron de Fernandes et al. (2012), siendo de 4,8 $\pm 0,2 \%$ o para el primero y de $10,1 \pm 0,2 \%$ o para el segundo, considerando una incertidumbre adicional de $0,5 \%$, con el fin de dar cuenta del efecto del tamaño corporal (Passey et al. 2005). A su vez, basándose en estos mismos estudios experimentales, se consideró el aporte diferencial de los macronutrientes y, por lo tanto, una contribución del $74 \pm 4 \%$ del carbono proteico en el carbono del colágeno, siendo el restante $26 \%$ proveniente de carbohidratos y lípidos (Fernandes et al. 2012). Destacamos que este aspecto es particularmente relevante a la hora de analizar la importancia de recursos de origen vegetal en la dieta, como es el caso de la presente investigación. El fraccionamiento entre la dieta y el colágeno óseo humano de los valores $\delta^{15} \mathrm{~N}$ considerado fue de 5,5 $\pm 0,5 \%$ o (Fernandes 2016).

Finalmente, con el fin de acotar la incertidumbre, incluimos una fuente de información previa. Consideramos una ingesta de proteína en un nivel de entre el 5 y el 45\%, basándonos en los estudios existentes sobre la cantidad adecuada que debe incluirse en la dieta (Fernandes et al. 2014; Otten et al. 2006).

\section{Resultados}

De las 19 piezas óseas analizadas, solo 12 superaron el protocolo para la extracción de colágeno, obteniéndose a su vez, porcentajes aceptables de colágeno (a excepción del individuo 19 -Tabla 2- del sitio Flores 1, que mostró un porcentaje de recuperación menor al 1\%) y una relación de $\mathrm{C} / \mathrm{N}$ satisfactoria (rango de aceptación entre 2,9 y 3,6 en De Niro 1985). Las muestras excluidas corresponden principalmente a los sitios Muyuna y Banda de Perchel, ambos correspondientes al PDRI. En el caso de la extracción de bioapatita, no se pudieron obtener resultados en dos individuos del sitio Muyuna, dado que no se contó con material suficiente para la medición. El resto no contó con suficiente colágeno para la medición isotópica, aunque sí se obtuvieron mediciones en la fracción inorgánica de las piezas óseas. Los resultados obtenidos en este conjunto deben ser tomados con cautela, dada la pérdida de colágeno mencionada. En la Tabla 2 se detallan los resultados obtenidos de todos los sitios analizados (media total y desvío estándar $=$ $\delta^{13} \mathrm{C}_{\mathrm{co}}-10,3 \pm 1,1 \% ; \delta^{15} \mathrm{~N}+10,7 \pm 1,4 \% ; \delta^{13} \mathrm{C}_{\mathrm{ap}}-4,8 \pm$ $1,1 \%$ o). Los valores medios del sitio Flores 1 fueron $\delta^{13} \mathrm{C}_{\mathrm{co}}-10,2 \pm 1,5 \%$; $\delta^{15} \mathrm{~N}+11,0 \pm 1,1 \%$ y y $\delta^{13} \mathrm{C}_{\mathrm{ap}}-4,0$ $\pm 1,3 \%$. Por su parte, los valores medios de Muyuna fueron $\delta^{13} \mathrm{C}_{\mathrm{co}}-9,9 \pm 1,1 \% ; \delta^{15} \mathrm{~N}+11,4 \pm 0,2 \% ; \delta^{13} \mathrm{C}_{\mathrm{ap}}$ $-5,1 \pm 0,5 \%$. Por último, para el caso de los individuos hallados en Pucará de Tilcara los valores medios fueron $\delta^{13} \mathrm{C}_{\mathrm{co}}-11,1 \pm 0,6 \%$; $\delta^{15} \mathrm{~N}+8,8 \pm 1,2 \%$ y $\delta^{13} \mathrm{C}_{\text {ap }}$ $-5,7 \pm 0,9 \%$.

A partir de los gráficos bivariados que cruzan la información isotópica de todos los sistemas considerados (Figuras 2 y 3 ), resulta evidente que no existen diferencias en las distribuciones de valores al considerar el agrupamiento de los individuos por sitios arqueológicos de procedencia y, por lo tanto, por períodos cronológico. Es posible señalar que los valores isotópicos de nitrógeno más bajos, generalmente asociados a una menor ingesta de recursos animales, se encuentran entre los individuos de la ocupación incaica del Pucará de Tilcara, como también en uno de los individuos del sitio Flores 1. Es decir, individuos asociados al comienzo y el final del lapso considerado. No obstante, al comparar los valores medios del carbono en bioapatita, notamos que tienden a ser cada vez más bajos con el paso del tiempo, aunque el tamaño de la muestra nos impida afirmar algún grado de significancia, sobre todo por lo anteriormente señalado respecto a las muestras degradadas del sitio Muyuna. Otro elemento para 
Tabla 2. Valores isotópicos de individuos humanos provenientes de los sitios arqueológicos analizados en este trabajo. $\mathrm{s} / \mathrm{d}=\sin$ datos.

Isotopic data of human individuals from archaeological sites analyzed in this work. $s / d=$ no data.

\begin{tabular}{|c|c|c|c|c|c|c|c|c|c|c|c|c|}
\hline $\mathrm{n}$ & Sitio & Sexo & Edad & Pieza ósea & AIE & $\begin{array}{c}\% \\
\text { colágeno }\end{array}$ & $\begin{array}{c}\delta^{13} \mathrm{C}_{\mathrm{co}}(\% o) \\
\text { V-PDB }\end{array}$ & $\begin{array}{c}\delta^{15} \mathrm{~N}(\%) \\
\quad \operatorname{AIR}\end{array}$ & $\mathrm{C} / \mathrm{N}$ & AIE & $\begin{array}{c}\delta^{/ 3} \mathrm{C}_{\mathrm{ap}}(\% o) \\
\text { V-PDB }\end{array}$ & Periodo asignado \\
\hline 1 & $\begin{array}{c}\text { Pucará de } \\
\text { Tilcara }\end{array}$ & I & A & $1^{\circ}$ costilla & 27518 & 16,8 & $-10,7$ & 9,9 & 3,2 & 28779 & $-5,5$ & Inca \\
\hline 2 & $\begin{array}{l}\text { Pucará de } \\
\text { Tilcara }\end{array}$ & I & A & $1^{\circ}$ costilla & 27510 & 17,6 & $-10,8$ & 7,5 & 3,2 & 28709 & $-5,0$ & Inca \\
\hline 3 & $\begin{array}{c}\text { Pucará de } \\
\text { Tilcara }\end{array}$ & I & A & $1^{\circ}$ costilla & 27513 & 16,1 & $-11,9$ & 9,1 & 3,3 & 28780 & $-6,7$ & Inca \\
\hline 4 & Muyuna & I & A & Cráneo & $\mathrm{s} / \mathrm{d}$ & $\mathrm{s} / \mathrm{d}$ & $\mathrm{s} / \mathrm{d}$ & $\mathrm{s} / \mathrm{d}$ & $\mathrm{s} / \mathrm{d}$ & 28708 & $-5,0$ & PDRI \\
\hline 5 & Muyuna & I & A & Cráneo & $\mathrm{s} / \mathrm{d}$ & $\mathrm{s} / \mathrm{d}$ & $\mathrm{s} / \mathrm{d}$ & $\mathrm{s} / \mathrm{d}$ & $\mathrm{s} / \mathrm{d}$ & 28773 & $-5,5$ & PDRI \\
\hline 6 & Muyuna & I & A & Cráneo & $\mathrm{s} / \mathrm{d}$ & $\mathrm{s} / \mathrm{d}$ & $\mathrm{s} / \mathrm{d}$ & $\mathrm{s} / \mathrm{d}$ & $\mathrm{s} / \mathrm{d}$ & 28772 & $-4,3$ & PDRI \\
\hline 7 & Muyuna & I & A & Cráneo & $\mathrm{s} / \mathrm{d}$ & $\mathrm{s} / \mathrm{d}$ & $\mathrm{s} / \mathrm{d}$ & $\mathrm{s} / \mathrm{d}$ & $\mathrm{s} / \mathrm{d}$ & 28711 & $-5,1$ & PDRI \\
\hline 8 & Muyuna & I & A & Cráneo & $\mathrm{s} / \mathrm{d}$ & $\mathrm{s} / \mathrm{d}$ & s/d & $\mathrm{s} / \mathrm{d}$ & $\mathrm{s} / \mathrm{d}$ & 29090 & $-5,0$ & PDRI \\
\hline 9 & Muyuna & $\mathrm{F}$ & A & $1^{\circ}$ vértebra & 27478 & 20,6 & $-8,8$ & 11,5 & 3,2 & $\mathrm{~s} / \mathrm{d}$ & $\mathrm{s} / \mathrm{d}$ & PDRI \\
\hline 10 & Muyuna & I & A & Atlas & 27476 & 7,0 & $-11,0$ & 11,2 & 3,4 & 28712 & $-5,7$ & PDRI \\
\hline 11 & Muyuna & $\mathrm{F}$ & A & falange & 27485 & 9,1 & $-10,0$ & 11,6 & 3,4 & $\mathrm{~s} / \mathrm{d}$ & $\mathrm{s} / \mathrm{d}$ & PDRI \\
\hline 12 & $\begin{array}{c}\text { Banda de } \\
\text { Perchel }\end{array}$ & I & A & Peroné & 27512 & 11,2 & $-9,9$ & 12,1 & 3,2 & 28704 & $-4,4$ & PDRI \\
\hline 13 & $\begin{array}{c}\text { Banda de } \\
\text { Perchel }\end{array}$ & I & A & Peroné & $\mathrm{s} / \mathrm{d}$ & $\mathrm{s} / \mathrm{d}$ & $\mathrm{s} / \mathrm{d}$ & $\mathrm{s} / \mathrm{d}$ & $\mathrm{s} / \mathrm{d}$ & $\mathrm{s} / \mathrm{d}$ & $\mathrm{s} / \mathrm{d}$ & PDRI \\
\hline 14 & $\begin{array}{c}\text { Banda de } \\
\text { Perchel }\end{array}$ & I & A & Peroné & $\mathrm{s} / \mathrm{d}$ & $\mathrm{s} / \mathrm{d}$ & $\mathrm{s} / \mathrm{d}$ & $\mathrm{s} / \mathrm{d}$ & $\mathrm{s} / \mathrm{d}$ & $\mathrm{s} / \mathrm{d}$ & $\mathrm{s} / \mathrm{d}$ & PDRI \\
\hline 15 & Flores & I & A & Omóplato & 27519 & 12,1 & $-8,1$ & 9,1 & 3,2 & 29086 & $-1,9$ & Formativo Tardío \\
\hline 16 & Flores & I & A & Omóplato & 27522 & 7,5 & $-11,1$ & 11,5 & 3,3 & 29106 & $-4,0$ & Formativo Tardío \\
\hline 17 & Flores & I & A & Omóplato & 27520 & 9,0 & $-11,7$ & 11,3 & 3,2 & 29110 & $-4,8$ & Formativo Tardío \\
\hline 18 & Flores & I & A & Cráneo & 27517 & 1,2 & $-10,8$ & 11,2 & 3,3 & 29109 & $-5,2$ & Formativo Tardío \\
\hline 19 & Flores & I & A & Cráneo & 27516 & 0,6 & $-9,3$ & 12,0 & 3,3 & 29092 & $-4,2$ & Formativo Tardío \\
\hline
\end{tabular}

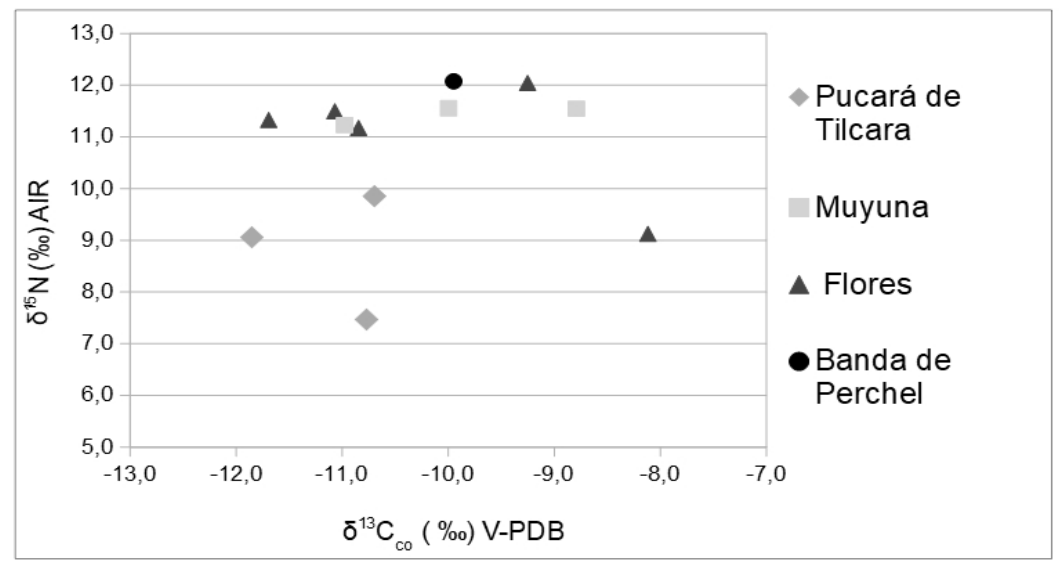

Figura 2. Valores $\delta 13 \mathrm{C}$ y $\delta 15 \mathrm{~N}$ del registro óseo humano procedentes de Flores 1 (Formativo Tardío); Muyuna, Banda de Perchel (periodo de Desarrollos Regionales I) y; Pucará de Tilcara (Inca).

$\delta^{13} \mathrm{C}$ and $\delta^{15} \mathrm{~N}$ values of human bone record from Flores 1 (Late Formative); Muyuna, Banda de Perchel (Regional Development Period I), and Pucará de Tilcara (Inca Period). 


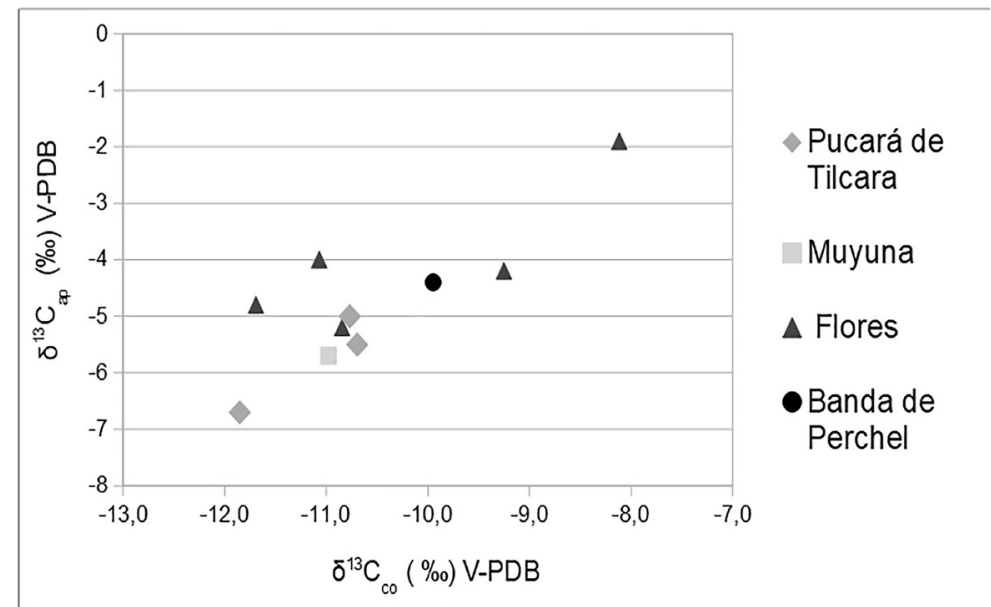

Figura 3. Valores $\delta^{13} \mathrm{C}$ en el colágeno y bioapatita del registro óseo humano procedentes de Flores 1 (Formativo Tardío); Muyuna, Banda de Perchel (periodo de Desarrollos Regionales I) y; Pucará de Tilcara (Inca).

$\delta^{13} C$ values of collagen and bioapatite of human bond record from Flores 1 (Late Formative); Muyuna, Banda de Perchel (Regional Development Period I), and Pucará de Tilcara (Inca Period)

tener en cuenta respecto a los valores isotópicos del carbono es que las mediciones de este elemento en ambas fracciones del registro óseo muestran una correlación positiva débil $\left(\mathrm{R}^{2}=0,7\right)$. Esto es esperable cuando la composición isotópica del componente energético es similar al proteico de la dieta o, como podría ocurrir en este caso, el componente vegetal (probablemente el maíz y/o amaranto) es tan importante que brinda la proteína necesaria para conformar el colágeno óseo.

\section{Aplicación del modelo bayesiano a los casos de estudio}

Utilizando una aproximación bayesiana que nos permite ponderar los aportes de los macronutrientes en las dietas finales de los individuos y, por lo tanto, evitar sesgos metabólicos en la interpretación de los resultados isotópicos, podemos inferir que todos los individuos analizados (10 poseen valores para los tres proxies paleodietarios considerados) tuvieron un aporte mayoritario de plantas $\mathrm{C}_{4}$ en su dieta (probablemente maíz y/o amaranto) (Tabla 3). Este aporte sobrepasa el $60 \%$ en todos los casos, existiendo incluso aportes por encima del $70 \%$ en dos individuos del sitio Flores 1. Resulta oportuno recordar que estos resultados se obtuvieron restringiendo el modelo mediante la inclusión de información previa, es decir, el supuesto fisiológico de un requerimiento proteico mínimo y necesario para la supervivencia humana. En el caso del aporte de los recursos vegetales $\mathrm{C}_{3}$ y del recurso Camelidae a la dieta de los individuos, estos fueron menores al $20 \%$ en la mayoría de los casos. En lo que respecta al recurso Camelidae, los que muestran el menor aporte se encuentran asociados tanto al sitio Flores 1 como el Pucará de Tilcara, aunque fueron los individuos de este último sitio los que mostraron valores $\delta^{15} \mathrm{~N}$ bajos en relación al resto del conjunto.

Aunque el número de muestras por sitio es pequeño, podemos señalar que en Flores 1 existieron diferentes patrones de consumo de alimentos, aunque, como ya señalamos, el alimento base ha sido el maíz (y/o el amaranto). Por ejemplo, el individuo 6 (Tabla 3 ) presenta un $87 \%$ de vegetales $\mathrm{C}_{4}$ en su dieta, siendo marginales el resto de los recursos, mientras que los individuos restantes muestran un aporte entre el 72 y el $63 \%$ de este recurso. A su vez, ese mismo individuo ostenta un aporte de $6 \%$ del recurso Camelidae, contrastando con el resto del conjunto, que posee entre un 16 y $18 \%$. Por su parte, los individuos provenientes del Pucará de Tilcara muestran bajas diferencias entre sí, siendo muy parejos los porcentajes de aportes de recursos en las dietas individuales. 
Tabla 3. Valores medios y desvío estándar (DS) de la contribución calórica (expresada en porcentajes) de cada conjunto de recursos en los individuos presentados en este

$$
\text { trabajo. }
$$

Mean values and standard deviation (SD) of the caloric contribution (in percentages) of each set of resources in the individuals presented in this work.

\begin{tabular}{cccccccc}
\hline \multicolumn{7}{c}{ Contribución calórica (\%) } \\
\hline \multicolumn{7}{c}{$\mathrm{C}_{4} /$ CAM } & \multicolumn{2}{c}{$\mathrm{C}_{3}$} & \multicolumn{2}{c}{ Camelidae } \\
\hline $\mathrm{n}$ & Sitio & Media & DS & Media & DS & Media & DS \\
\hline 1 & $\begin{array}{c}\text { Pucará de } \\
\text { Tilcara }\end{array}$ & 67,0 & 4,9 & 21,5 & 7,8 & 11,5 & 9,2 \\
& & & & & & \\
\hline 2 & $\begin{array}{c}\text { Pucará de } \\
\text { Tilcara }\end{array}$ & 72,1 & 4,1 & 22,8 & 5,8 & 5,1 & 4,9 \\
\hline 3 & $\begin{array}{c}\text { Pucará de } \\
\text { Tilcara }\end{array}$ & 61,0 & 4,4 & 31,5 & 6,4 & 7,5 & 6,7 \\
\hline 4 & Muyuna & 62,3 & 5,0 & 20,3 & 9,1 & 17,4 & 11,4 \\
\hline 5 & Banda de & 68,4 & 5,9 & 11,6 & 7,3 & 20,0 & 10,2 \\
\hline 6 & Perchel & & & & & & \\
\hline 7 & Flores 1 & 86,7 & 4,8 & 6,9 & 4,5 & 6,4 & 4,9 \\
\hline 8 & Flores 1 & 67,2 & 5,0 & 16,5 & 8,1 & 16,3 & 10,4 \\
\hline 9 & Flores 1 & 63,6 & 4,9 & 19,4 & 9,2 & 17,0 & 11,3 \\
\hline 10 & Flores 1 & 64,4 & 5,6 & 17,3 & 8,8 & 18,3 & 11,5 \\
\hline & Flores 1 & 72,0 & 5,4 & 10,0 & 6,8 & 18,0 & 9,4 \\
\hline
\end{tabular}

Figura 4. Box-plots correspondientes a la contribución calórica (expresada en porcentajes) de los tres grupos de recursos considerados para cada periodo cronológico. (A) $\mathrm{C}_{4} / \mathrm{CAM}$; (B) $\mathrm{C}_{3}$ y; (3) Camelidae.

Box-plots of the caloric contribution (expressed in percentages) of the three resource groups considered for each chronological period. (A) $C_{4} / C A M$; (B) $C_{3}$, and (3) Camelidae.

Tabla 4. Valores medios y desvío estándar (DS) de los sistemas isotópicos medidos en humanos considerando los períodos cronológicos de procedencia.

Mean values and standard deviation (DS) of isotopic systems measured in humans considering chronological periods.

\begin{tabular}{|c|c|c|c|c|c|c|c|c|c|c|c|c|c|}
\hline \multirow{3}{*}{$\begin{array}{l}\text { Periodo } \\
\text { asignado }\end{array}$} & \multirow{3}{*}{$\mathrm{n}$} & \multirow{2}{*}{\multicolumn{2}{|c|}{$\begin{array}{c}\delta^{13} \mathrm{C}_{\mathrm{co}}(\% \circ) \\
\text { V-PDB }\end{array}$}} & \multirow{2}{*}{\multicolumn{2}{|c|}{$\delta^{15} \mathrm{~N}(\% o)$ AIR }} & \multirow{2}{*}{\multicolumn{2}{|c|}{$\begin{array}{c}\delta^{13} \mathrm{Cap}(\%) \\
\text { V-PDB }\end{array}$}} & \multicolumn{6}{|c|}{ Contribución calórica (\%) } \\
\hline & & & & & & & & \multicolumn{2}{|c|}{$\mathrm{C}_{4} / \mathrm{CAM}$} & \multicolumn{2}{|c|}{$\mathrm{C}_{3}$} & \multicolumn{2}{|c|}{ Camelidae } \\
\hline & & Media & DS & Media & DS & Media & DS & Media & DS & Media & DS & Media & DS \\
\hline \multicolumn{14}{|l|}{ Formativo } \\
\hline Tardío & 9 & $-9,8$ & 1,2 & 10,8 & 1,1 & $-3,6$ & 1,3 & 72,3 & 8 & 12,1 & 7,9 & 15,7 & 10,2 \\
\hline PDR I & 2 & $-10,5$ & 0,7 & 11,7 & 0,6 & $-5,1$ & 0,9 & 67,3 & 6,3 & 14,4 & 8,5 & 18,4 & 11 \\
\hline PDR II & 20 & $-10,9$ & 1,0 & 10,1 & 0,8 & $-6,5$ & 0,8 & 61,2 & 6,7 & 22,8 & 10,3 & 16 & 11,9 \\
\hline Inca & 10 & $-11,0$ & 1,2 & 9,4 & 1,7 & $-5,6$ & 1,3 & 61,9 & 8,1 & 19,8 & 10,3 & 18,4 & 12,8 \\
\hline
\end{tabular}




\section{Tendencias generales para la región}

Como hemos señalado en el apartado metodológico, la aplicación del modelo de mezcla bayesiano FRUITS solo consideró a aquellos individuos que fueron medidos mediante distintos sistemas isotópicos, con lo cual no podemos incluir en la misma discusión aquellos casos donde esto no ha sido posible. Por lo tanto, si bien no podemos aplicar el modelo a todos los casos disponibles Quebrada de Humahuaca, sí podemos incluir los datos generados hasta el momento para establecer las tendencias que pudieron prevalecer en la región, considerando los valores promedios de cada sistema isotópico. En la Tabla 4 se detalla esta información extraída de Amman et al. 2014; Lynch Ianniello et al. 2018 y este trabajo para el periodo Formativo (sitios Til 22 y Flores 1); este trabajo para el PDR I (sitios Muyuna y Banda de Perchel); Amman et al. 2014, Killian Galván et al. 2016b y Olivera y Yacobaccio 1999 para el PDR II (sitios Yacoraite, Los Amarillos, La Huerta y Huachichocana III); finalmente Gheggi et al. (2018) y este trabajo para el periodo Incaico (sitios Esquina de Huajra y Pucará de Tilcara). El número de individuos en total es de 41 .

Al aplicar el modelo de mezcla bayesiano de reconstrucción paleodietaria (Figura 4), hallamos que el periodo Formativo Tardío ostenta el porcentaje más alto de plantas $\mathrm{C}_{4} / \mathrm{CAM}$ en la dieta $(72 \%)$, siendo este aporte descendente a lo largo del tiempo (PDRI = $67 \%$; PDRII $=61 \%$; Inca $=62 \%)$. La tendencia contraria se halla al evaluar el aporte de plantas $\mathrm{C}_{3}$ (Formativo Tardío $=12 \%$; PDRI $=14 \%$; PDRII $=$ $23 \%$; Inca $=20 \%$ ). Con respecto al recurso Camelidae, no es posible establecer una tendencia clara, aunque aparece como una constante que su aporte a la dieta fue secundario en relación con el componente vegetal (Formativo Tardío $=16 \% ;$ PDRI $=18 \%$; PDRII $=$ $16 \%$; Inca $=18 \%$ ). Es decir, el maíz o en su defecto el amaranto, dado que no podemos discriminar el aporte específico de cada uno dentro de las plantas $\mathrm{C}_{4}$, durante los periodos coincidentes con la agudización de la conflictividad social parecieran ser relativamente menos importantes, aunque su relevancia sigue siendo indiscutible.

Por su parte, los análisis intrasitio realizados con este modelo muestran una escasa variación en el sitio Formativo Til 20 y en Yacoraite, ambos con al menos tres individuos medidos y con cronologías asociadas al Formativo Tardío y a los Desarrollos Regionales II-Inca, respectivamente. Esta escasa diferenciación ya ha sido registrada en el sitio Los Amarillos, incluso comparándose los resultados provenientes de diferentes sectores del sitio arqueológico (Killian Galván 2018; Killian Galván et al. 2016b). Distinto es el caso de Esquina de Huajra, sitio plenamente incaico, donde aparecen diversos aportes calóricos de los diferentes recursos. Esto puede significar dos cosas. O bien convivieron patrones de consumo que respondieron a diferentes adscripciones sociales o se trata de un sitio donde convergieron individuos de diferentes espacios geográficos, cuyos alimentos poseían una composición isotópica también diferente.

\section{Discusión}

Partiendo de una concepción histórica del cambio, según la cual las contingencias inherentes a la práctica generan trayectorias únicas y configuraciones sociales siempre nuevas, entendemos que las formas y modalidades de consumo son consecuencia de los procesos sociales, ambientales y políticos que allí tuvieron lugary, por lo tanto, no extrapolables al resto del mundo andino. Bajo este marco es que comprendemos la evidencia isotópica. La Quebrada de Humahuaca es en la actualidad una de las regiones con mayor número de investigaciones basadas en el análisis de isótopos estables en restos esqueletales del NOA, lo cual permite inferir tendencias de largo tiempo en los patrones de consumo de alimentos. Estos análisis han sido aplicados en series esqueletales no solo de sociedades agropastoriles, sino también en las primeras ocupaciones cazadoras-recolectoras, como es el caso de sitios paradigmáticos como Inca Cueva 4 y Huachichocana III (Olivera y Yacobaccio 1999) durante el Holoceno Temprano. Los investigadores que llevaron a cabo estos estudios concluyeron que los valores isotópicos registrados para el individuo de Huachichocana III, concordaban con la expectativa generada por el registro arqueológico (Olivera y Yacobaccio 1999), es decir, el consumo de camélidos y roedores, junto con plantas CAM como Opuntia sp. y Tillandsia sp. halladas en el sitio arqueológico (Yacobaccio 1997). Entonces, desde las primeras ocupaciones humanas, la composición isotópica de los individuos evidenció la importancia de vegetales en la dieta.

No obstante, para el Holoceno Medio y comienzos del Tardío los hallazgos de restos esqueletales humanos en elárea son escasos. Nuevamente, contamos con valores isotópicos obtenidos en restos óseos hallados en Huachichocana III, con una cronología de 3400 años AP (presentando los valores $\delta^{13} \mathrm{C}_{\text {co }}$ $-17,1 \% \circ ; \delta^{15} \mathrm{~N}+10,5 \% \circ ; \delta^{13} \mathrm{C}_{\text {ap }}-8,7 \%$ ). Si aplicamos a este individuo el modelo bayesiano utilizado en este trabajo, el porcentaje de plantas C4 o CAM en la dieta es importante (lo cual es llamativo, dada la ausencia de macrorrestos de maíz o amaranto en el registro arqueológico), pero no 
llega a ser el más relevante respecto al resto de los recursos $\left(\mathrm{C}_{4} / \mathrm{CAM}=35 \% ; \mathrm{C}_{3}=57 \%\right.$ y Camelidae $=$ 9\%). Debemos esperar hasta los hallazgos en Til 20 y Flores 1, es decir, las ocupaciones del comienzo del primer milenio de la era cristiana, para tener elementos que permitan caracterizar la dieta del Formativo, la cual como hemos señalado, contrasta con lo inferido para el individuo de Huachichocana III, pues el porcentaje del aporte calórico del maíz y/o amaranto hallado en esos sitios es mucho mayor. Un elemento para tener en cuenta es que la agricultura desarrollada en cercanías al Río Huasamayo, en el departamento de Tilcara y, por lo tanto, en vinculación con los sitios Til 1 y Flores 1, han mostrado relaciones estrechas con los sitios San Francisco de comienzos del primer milenio, ubicados en las Yungas de Jujuy (González 2011). Los análisis de isótopos estables realizados sobre restos óseos humanos procedentes de dichos sitios han mostrado la relevancia de recursos $\mathrm{C}_{4} \mathrm{y} / \mathrm{o}$ CAM en la dieta en la mayoría de los individuos (Ortiz y Killian Galván 2016). Con esto no queremos afirmar que esos contactos fueron acompañados siempre de semejanzas en la valoración de ciertos artículos culinarios o elecciones de consumo, pues los indicadores vinculados a San Francisco se encuentran ampliamente distribuidos, llegando a la Puna (sitios como Coch39 en Salinas Grandes y Matancillas en San Antonio de los Cobres) y hasta San Pedro de Atacama (Tulor). Es decir, que se trató de un estilo circulado y replicado por comunidades con economías muy diversas. Sin embargo, entendemos que en Quebrada de Humahuaca, a raíz de las posibilidades que ofrecía su ambiente y el contexto social local, pudo existir una fuerte influencia e intercambio entre las sociedades que ocuparon estas áreas, no solo en lo referente a estrategias tecnológicas, sino en la elección de recursos consumidos.

Por lo tanto, si bien no contamos con datos suficientes para caracterizar el pasaje de las sociedades cazadoras-recolectoras a aquellas formativas en términos paleodietarios, podemos postular que la explotación de maíz estuvo bien establecida entre los cultivos de fondo de cuenca ya en el Formativo Tardío. Este cereal pudo consolidarse como un recurso que, por su versatilidad, disponible a lo largo del año gracias a la implementación de algún tipo de almacenaje, habría garantizado la alimentación de las unidades domésticas. No obstante, no podemos descartar que este rol protagónico también pudo jugarlo el amaranto, de amplias cualidades nutricionales, pero menos evidente en cuanto a su presencia en el registro arqueológico del área. Es decir, que si bien la agricultura de la época probablemente no estuvo acompañada de inversiones materiales como las que se desarrollarían posteriormente para irrigar los campos alejados del fondo de valle (escenario especulativo que será difícil de superar dado que la evidencia del fondo de valle ha sido sepultada por aluviones y alterada por la ocupación continua), el maíz y/o el amaranto pudieron integrarse rápidamente como alimentos base. Coincidimos con Lema (2014) y Quesada et al. (2012), en que la agricultura del primer milenio de la Era pudo ser relativamente sencilla en la faz arquitectónica, pero implicar una sofisticada tecnología en términos de conocimiento de cultivos, manejo de suelos, agua y fertilización. Estos resultados concuerdan, a su vez, con las conclusiones de Alfonso-Durruty et al. (2017), quienes afirman que la adopción y consumo de maíz no fue uniforme en los Andes meridionales, pues en otras regiones el lugar del maíz como alimento base se consolidó muy tardíamente. Basta detenerse en las investigaciones de las ocupaciones del Valle de Moquegua, sur de Perú, durante el Horizonte medio (500 - 1000 AD). Allí las colonias del Estado Tiwanaku, Río Muerto y Omo, produjeron cantidades suficientes de maíz no solo como para convertirse en el alimento base de la población, sino incluso, para exportarlo para su usufructo en el centro del poder político, donde las condiciones ambientales eran adversas para la producción cerealera masiva (Somerville et al. 2015). Debe decirse, sin embargo, que la importancia del maíz no llegó a ser equiparable a la que tuvo lugar en sitios del contemporáneo Estado de Wari, p.ej., Conchopata en los Andes centrales (Finucane et al. 2006). Paralelamente, un estudio de diferentes sitios asociados a la cultura Chiribaya, en el Valle del Río Osmore, costa peruana (Tomczak 2003), muestra la convivencia de economías afianzadas en la pescadería o el maíz, incluso durante el periodo Intermedio Tardío (PIT; 1000 - 1450 AD). Es decir, una especialización económica que reflejaría las condiciones ecológicas locales, siendo la altitud y la cercanía al mar las variables determinantes. A su vez, la diversidad de estrategias económicas también se vislumbra en el Norte árido chileno, donde si bien se ha registrado un aumento progresivo del consumo de maíz desde el periodo Formativo (1500 AC - 400 DC) hasta el PIT, el consumo de proteína marítima se mantiene como una constante en poblaciones costeras o cercanas a la costa (Mora et al. 2017; Pestle et al. 2015). Quizá uno de los casos más llamativos y que pone de manifiesto la falta de uniformidad durante el PIT, es el caso de Quillagua, oasis que ofició de nodo entre las poblaciones de la costa y las tierras altas andinas. Allí, la importancia de los recursos marítimos, teniendo al maíz como un complemento, 
no se explica por factores meramente ecológicos, al encontrarse a una distancia de $70 \mathrm{~km}$ respecto a la costa, sino probablemente a una identificación por parte de los locales con la Cultura Pica-Tarapacá, asociada con la explotación marítima (Cementerio Pica 8) y con la Cultura Atacama, de agricultores y pastores (Cementerio Quitor 6) (Santana-Sagredo et al. 2016).

Retomando lo ocurrido en Quebrada de Humahuaca, el escenario hasta aquí delineado pudo cambiar sensiblemente hacia los ca. 1250 AD, en el transcurso del denominado periodo de Desarrollos Regionales II, cuando los grupos que ocuparon esta Quebrada establecieron estrategias económicas diferentes, con una mayor intensificación, evidenciado por la inversión en tecnología en riego, así como parapetos y puestos para el pastoreo en altura. El correlato dietario pudo ser una mayor inclusión de tubérculos y pseudocereales como la quínoa, característicos de los cultivos de altura, aunque como han mostrado nuestros resultados, el maíz se mantuvo hasta incluso la llegada del Inca como el recurso fundamental de la alimentación. En cierto modo, podría tratarse del correlato a las tendencias identificadas en los análisis zooarqueológico, como ser la consolidación de la trashumancia pastoril y la disminución de la caza (Madero 1993), como también la explotación de carácter secundario de los rebaños (Mercolli et al. 2014). No obstante, no hemos logrado identificar una disminución de la importancia del componente cárnico en la dieta, sino su rol periférico en la alimentación cotidiana (contribuyendo en promedio siempre en el orden del $20 \%$ ) como una constante a lo largo del tiempo.

$\mathrm{Si}$ el componente vegetal $\mathrm{C}_{4}$ que primó en la Quebrada de Humahuaca fue el maíz, su adopción rápida como recurso alimenticio de primer orden y, por lo tanto, la producción cerealera, no aparece como la respuesta a la necesidad de generar un recurso capaz de sustentar una población más numerosa ya sea por crecimiento poblacional o por su concentración en la quebrada troncal, pues su consumo masivo precede a la constitución de los grandes conglomerados habitacionales del PDR II. Así, es posible pensar que las garantías que ofrece la Quebrada de Humahuaca para cosechas exitosas pudieron posicionar al maíz como un recurso central para la población, capaz de dictar el tempo del calendario productivo. Durante este extenso periodo el maíz pudo ser un "super alimento cultural" (sensu Jelliffe 1967), un recurso que, con una fuerte carga simbólica, proporcionó la mayor parte del valor calórico de la dieta. No obstante, existen evidencias que muestran un aumento en la diversidad de morfotipos en el maíz en los Andes meridionales
(Oliszewski 2012 y Vidal-Elgueta et al. 2016), sobre todo en las ocupaciones más tardías, lo cual podría interpretarse como un correlato concreto de un aumento en el consumo efectivo. Entendemos que al menos en el caso de Quebrada de Humahuaca esto no fue así. La búsqueda e introducción de variedades diferentes, como señala Oliszewski (2012), podría responder a la satisfacción de demandas que superan el plano estrictamente nutricional, pues si bien las diferentes razas permitieron contar con cosechas en diferentes momentos del año, lo cual lo convierte en un recurso altamente redituable en términos económicos, la amplia gama de fenotipos que caracteriza a esta planta garantizó su uso en contextos culinarios y rituales diversos. Las unidades alimentarias (sensu Contreras Hernández 1993), es decir, las diferentes preparaciones y formas de presentar los alimentos base, pudieron involucrar la búsqueda de diversidad de razas, no solo con el fin de maximizar los rindes agropecuarios o nutricionales, sino para lograr los matices propios de un alimento lujoso (sensu Hastorf 2003). Por ejemplo, como se ha registrado en documentos etnohistóricos andinos, las bebidas fermentadas utilizaban distintas razas de maíz, siendo importante su procedencia desde largas distancias (Hastorf 2003). En este sentido, la proliferación de escenarios de congregación con indicios de comensalismo político, hecho asociado a las reorganizaciones sociales características del periodo de Desarrollos Regionales (Nielsen 2006b), pudo dar lugar a la búsqueda de nuevas unidades alimentarias, involucrando modalidades alternativas de presentación y consumo de un recurso cotidiano como fuera el maíz. Incluso, como ha propuesto Leibowicz (2012) a partir del hallazgo de evidencias relativas a la producción de chicha, las condiciones naturales que se presentan en la región, particularmente convenientes para el crecimiento del maíz, pudieron dar lugar a una producción y consumo mucho más frecuente de este brebaje, no restringido a eventos especiales.

Un último aspecto atañe a la distribución. De acuerdo con Raffino (2007), los cambios socioeconómicos hasta aquí enumerados pudieron resultar en una mayor desigualdad en las prácticas de consumo de alimentos al interior de los grupos sociales. Otros, sin embargo, han propuesto que los procesos de integración de este periodo operaron en base a principios corporativos o comunales (Acuto 2007; Nielsen 2006b), que no implicaron un acceso diferencial a los recursos. La propuesta de Belotti López de Medina et al. (2016), mediante la inferencia de patrones de consumo de fauna en Quebrada de Humahuaca refuerza dicha perspectiva. Considerando lo anterior, a partir de los datos obtenidos, no 
hallamos un correlato isotópico a las diferencias en los estilos de vida que pudieron darse a partir de los cambios en la organización sociopolítica planteados para la región. Al menos a partir de la jerarquización de estos grandes conjuntos de alimentos (Vegetales $\mathrm{C}_{3}$, vegetales $\mathrm{C}_{4} / \mathrm{CAM}$ y Camélidos) no podemos inferir un acceso diferencial a los recursos. Nuestros análisis intrasitio, tanto en las ocupaciones más tempranas como en las tardías no muestran a la alimentación como un componente altamente variable. Solo en el sitio Esquina de Huajra, de adscripción incaica, se ha registrado una diferenciación a nivel de macroconjuntos, pero como ya hemos mencionado, es probable que este fuera un sitio de confluencia, con individuos en tránsito desde diferentes puntos de la región. La diversidad encontrada en la composición isotópica de los ejemplares de camelidae allí hallados (Mengoni Goñalons 2014) apoyaría este escenario. Pese a ello, no descartamos que algunas formas particulares de consumo, significativas socioculturalmente, pudieron operar en el pasado como marcadores de estatus, pero las mismas no son distinguibles mediante la vía metodológica propuesta en este trabajo, siendo necesaria la complementación de distintas líneas de evidencia para su identificación.

\section{Conclusiones}

La reconstrucción de las trayectorias del maíz entre las sociedades prehispánicas andinas es un tópico que se ha visto notablemente enriquecido a partir de la aplicación de metodologías basadas en el análisis de isótopos estables en el registro esqueletal humano, pues ha permitido comprender de una manera eficiente el vínculo entre la producción, la distribución y el consumo del cereal, al ser una línea de investigación independiente y con una escala de resolución individual. En el caso de su aplicación en los contextos arqueológicos de Quebrada de Humahuaca prehispánica, nos ha permitido fortalecer la hipótesis de que la alimentación ha reposado en el maíz (y/o el amaranto) como alimento principal, siendo secundario el recurso animal. También ha demostrado, que esto ya habría ocurrido en las economías del Formativo Tardío, antes de las inversiones tecnológicas asociadas a los periodos posteriores. Por lo tanto, a diferencia de otras regiones del sur andino, su relevancia no se incrementó como el resultado de una política de Estado, sino que fue el alimento base fundamental de la población local durante al menos 1.500 años.

Agradecimientos: Agradecemos al personal del Instituto Interdisciplinario Tilcara (FFyL-UBA), en particular a Armando Mendoza y al personal de la Reserva Arqueológica por colaborar en las tareas de laboratorio; a Estela Ducós y Nazareno Piperizza por su trabajo en el Laboratorio de Biogeoquímica de INGEIS; a Celeste T.Samec por sus valiosos aportes a la hora de interpretar los valores; a Carlos Bellotti y María de las Victorias Villa por sus recomendaciones bibliográficas; a Andrés E. Duhour por su asesoramiento cartográfico; y a Gladys A. Galván por su colaboración en las tareas de cuidado familiar, imprescindible para poder llevar a cabo la escritura de este artículo. Este trabajo se vio enriquecido por la evaluación de tres investigadores/as anónimos/as, un agradecimiento particular para ellos/ ellas.

\section{Referencias Citadas}

Acuto, F.A. 2007. Fragmentación vs. integración comunal: repensando el Período Tardío del Noroeste Argentino. Estudios Atacameños 34:71-95.

Albeck, M.E. 1992. El ambiente como generador de hipótesis sobre dinámica sociocultural prehispánica en la Quebrada de Humahuaca. Cuadernos 3:95-106.

Alfonso-Durruty, M., A. Troncoso, P. Larach, C. Becker y N. Misarti 2017. Maize (Zea mays) consumption in the southern andes (30-31 S. Lat): Stable isotope evidence (2000 BCE-1540 CE). American Journal of Physical Anthropology 164 (1):148-1.

Ammann, M.G., O.J. Mendonça, N.I. Merlo, M.A. Bordach y R.H. Tykot 2014. Stable isotopes ( $\delta 13 \mathrm{C}$ and $815 \mathrm{~N}$ ) from North West Argentina: paleodietary implications. Open Journal of Archaeometry 2 (1).

Belotti López de Medina, C.R., L. López y C. Otero 2016. At the feet of the fortress. Late Pre-hispanic Period (ca. AD 1200-1500) archaeofaunal assemblages analysis from Residential Unit (RU1), Pukara de Tilcara (Jujuy, Argentina). PLoS ONE 11 (10):e0163766.

Bray T. 2003. The commensal politics of early states and empires. En The Archaeology and Politics of Food and Feasting in Early States and Empires, editado por T. Bray, pp. 1-13. Kluwer Academic Publishers, New York.

Burger, R.N. y N.J. Van Der Merwe 1990. Maize and the origin of Highland Chavín civilization: An isotopic perspective. American Anthropologist 92:85-95.

Cabrera, A.L.1976. Regiones fitogeográficas argentinas Enciclopedia Argentina de Agricultura y Jardinería, editada por W.F. Kugler, Tomo 2, Fascículo 1. pp. 1-85. Editorial Acme, Buenos Aires.

Contreras Hernández, J. 1993. Antropología de la Alimentación. Eudema, Madrid.

Craig, H. 1957. The natural distribution of radiocarbon and the exchange time of carbon dioxide between atmosphere and sea. Tellus 9 (1):1-17. 
De Niro, M. 1985. Postmortem preservation and alteration of in vivo bone collagen isotope ratios in relation to palaeodietary reconstruction. Nature 317:806-809.

De Molina, C. 1959 [1575]. Ritos y Fábulas de los Incas. Editorial Futuro, Buenos Aires.

Fernandes, R. 2016. A simple (R) model to predict the source of dietary carbon in individual consumers. Archaeometry 58 (3):500-512.

Fernandes, R., A.R. Millard, M. Brabec, M.J. Nadeau, P.M. Grootes 2014. Food reconstruction using isotopic transferred signals (FRUITS): a Bayesian model for diet reconstruction. PLoS One: http://dx.doi.org/10.1371/journal.pone.0087436.

Fernandes, R., M.J. Nadeau y P.M. Grootes 2012. Macronutrientbased model for dietary carbon routing in bone collagen and bioapatite. Archaeological and Anthropological Sciences 1-11.

Fernández Distel, A. 1980. Los fechados radiocarbónicos de la arqueología de la provincia de Jujuy. Fechas radiocarbónicas de la cueva CH III de Huachichocana, Tiuiyaco e Inca Cueva. Radiocarbono en Arqueología 1 (4/5).

Finucane, B., P.M. Agurto y W.H. Isbell 2006. Human and animal diet at Conchopata, Peru: stable isotope evidence for maize agriculture and animal management practices during the Middle Horizon. Journal of Archaeological Science 33 (12):1766-1776.

Garvie-Lok, S.J., T.L. Varney y M.A. Katzenberg 2004. Acetic acid treatment of bone carbonate: the effects of treatment time and solution concentration. Journal of Archaeological Science 31:763-776.

Gheggi, M.S. y V.I. Williams 2013. New data on food consumption in pre-hispanic populations from Northwest Argentina (ca. 1000$1550 \mathrm{AD}$ ): the contribution of carbon and nitrogen isotopic composition of human bones. Journal of Anthropology. http:// dx.doi.org/ 10.1155/2013/258190.

Gheggi, M.S., V.I. Williams y M.B. Cremonte 2018. The impact of the Inca Empire in Northwest Argentina: Assessment of health status and food consumption at Esquina de Huajra (Quebrada de Humahuaca, Argentina). International Journal of Osteoarchaeology 28 (3):274-284.

González, N. M. 2011. Aprovechamiento del espacio agrícola en la cuenca del río Huasamayo (departamento Tilcara, Provincia de Jujuy, Argentina). La Zaranda de Ideas: Revista de Jóvenes Investigadores en Arqueología 7:97-113.

Greco, C. y C. Otero 2016. Chronology of settlements with pre-Inca and Inca occupations superimposed. The case of Pucará de Tilcara (Humahuaca Gorge, Argentina). Archaeometry 58 (5):848-862.

Haber, A. 2006 Agrícola est quem domus demonstrat. En Contra la tiranía tipológica en Arqueología: una visión desde Sudamérica, editado por C. Gnecco y C. Langebaek, pp. 7798. Universidad de los Andes, CESO, Bogotá.

Hastorf, C.A. 1985. Dietary reconstruction in the Andes: A new archaeological technique. Anthropology Today 1 (6):19-21.

Hastorf, C.A. 2003 Andean Luxury Foods: special food for the ancestors, the deities and the elite. Antiquity 77:110-119.

Ikehara, H.C., J.F. Paipay y K. Shibata 2013. Feasting with Zea mays in the middle and late formative north coast of Peru. Latin American Antiquity 24 (2):217-231.

Jelliffe, D.B. 1967. Parallel food classifications in developing and industrialized countries. The American Journal of Clinical Nutrition 20 (3):279-281.
Killian Galván, V.A. 2018. Models for paleodietary research: Three case-studies from arid and semi-arid environments in Northwest Argentina. Journal of Archaeological Science: Reports 18:608-616.

Killian Galván, V.A., C.T. Samec y H.O. Panarello 2016a. When maize is not the first choice: advances in paleodietary studies in the Archaeological Site Río Doncellas (Jujuy, Argentina). Anthropological Review 79 (3):265-279.

Killian Galván, V.A., G. Sanmartino, V. Castellano, V. Seldes y L. G. Marban 2015. Estudios de isótopos estables en huertas familiares actuales de la Quebrada de Humahuaca (Jujuy, Argentina). Su potencial aporte a los estudios paleodietarios del Noroeste argentino. Revista del Museo de Antropología 8 (2):107-118.

Killian Galván, V.A. V. Seldes y A.E. Nielsen 2016b. Inferencia paleodietaria en el sitio arqueológico Los Amarillos (Quebrada de Humahuaca, Jujuy, Argentina). Relaciones de la Sociedad Argentina de Antropología 41 (1):79-99.

Korstanje, A. 2015. Andenes en los Andes: paisajes agrícolas tardíos sin maíz. En Racionalidades Campesinas en los Andes del Sur. Reflexiones en Torno al Cultivo de la Quinoa y Otros Vegetales Andinos, editado por P. Cruz, R. Joffre y T. Winkel, pp. 21-57. Editorial de la Universidad Nacional de Jujuy, Jujuy.

Krapovickas, P. 1983. Hallazgos incaicos en Tilcara y Yacoraite (una reinterpretación). Relaciones de la Sociedad Argentina de Antropología 14:67-80.

Lavallée, D., M. Julien, C. Karlin, L.C. García, D. Pozzi-Escot y M. Fontugne 1997. Entre desierto y quebrada. Primeros resultados de las excavaciones realizadas en el abrigo de Tomayoc (Puna de Jujuy, Argentina). Bulletin de l'Institut Français d'Études Andines 26 (2):141-176.

Lantos, I., J.E. Spangenberg, M.A. Giovannetti, N. Ratto y M. S. Maier 2015. Maize consumption in pre-Hispanic south-central Andes: chemical and microscopic evidence from organic residues in archaeological pottery from western Tinogasta (Catamarca, Argentina). Journal of Archaeological Science 55:83-99.

Leibowicz, I. 2012. Ideología y Espacio: Conquista Inka en la Quebrada de Humahuaca, Jujuy, Argentina. Revista Chilena de Antropología 25:409-422.

Lema, V. 2014. Boceto para un esquema: domesticación y agricultura temprana en el Noroeste argentino. Revista Española de Antropología Americana 44 (2):465-494.

Leoni, J.B., J. Sartori, G. Fabron, A. Hernández y G. Scarafia 2012. Aportes al conocimiento de las sociedades aldeanas del período Temprano en la Quebrada de Humahuaca: una visión desde Antumpa. Intersecciones en Antropología 13 (1):117-131.

Lynch Ianniello, I., O.J. Mendonça, M.A. Arrieta, L. Bernardi y M.A. Bordach 2018. Exploring dietary trends in late Holocene populations from Northwest Argentina: Insights from new data on stable isotope analysis $(\delta 13 \mathrm{C}$ and $\delta 15 \mathrm{~N})$. Journal of Archaeological Science: Reports 18:617-627.

Madero, C. 1993. Explotación faunística, tafonomía y economía en Humahuaca antes y después de los Yupanki. En Inka: Arqueología, Historia y Urbanismo del Altiplano Andino, editado por R.A. Raffino, pp. 145-168. Corregidor, Buenos Aires.

McCrea, J.M. 1950. On the isotopic chemistry of carbonates and a paleotemperature scale. Journal of Chemical Physics 18:849-857.

Mendonça, O., M.A. Bordach, M. Grosso y L. Dalerba 1991. Nuevas evidencias del período agroalfarero temprano en Quebrada 
de Humahuaca. Los hallazgos del sitio Til 20 (Tilcara, Jujuy). Comechingonia 7:31-45.

Mengoni Goñalons, G.L. 2007. Camelid managment during Inca times in N.W. Argentina: models and archaeozoological indicators. Anthropozoologica 42 (2):129-141.

Mengoni Goñalons, G.L. 2014. Isótopos estables en camélidos del período tardío del noroeste de Argentina (NOA): estrategias de uso y manejo de rebaños. Revista Chilena de Antropología 30:68-75

Mercolli, P. 2005. Tendencias en las estrategias de caza durante el Período de Desarrollos Regionales en la Quebrada de Humahuaca. Estudios Sociales del NOA 8 (8):188-218.

Mercolli, P., M. Vazquez y K. Menacho 2014. Pucará de Huichairas en el contexto regional de la Quebrada de Humahuaca (Departamento de Tilcara, Prov. de Jujuy). En Integración de Diferentes Líneas de Evidencia en la Arqueología Argentina, editado por G. Cassiodoro, A. Re y D. Rindel, pp. 41-58. Aspha, Buenos Aires.

Merlo, N.I., O.J. Mendonça, M.A. Bordach y M.S. Ruiz 2005. Vida y muerte en el Pucará de Yacoraite: Estudio de osteología humana. Cuadernos de la Facultad de Humanidades y Ciencias Sociales. Universidad Nacional de Jujuy 29:113-142.

Métraux, A. 1972. Los Incas. Centro Editor de América Latina, Buenos Aires.

Mora, A., B.T. Arriaza, V.G. Standen, C. Valdiosera, A. Salim y C. Smith 2017. High-resolution palaeodietary reconstruction: Amino acid $813 \mathrm{C}$ analysis of keratin from single hairs of mummified human individuals. Quaternary International 436:96-113.

Muñoz, A. y P. Stenborg 1999. Conclusions. En Masked Histories: A Re-Examination of the Rodolfo Schreiter Collection from North Western Argentina, editado por P. Stenborg y A. Muñoz, pp. 279285. Göteborgs Etnografiska Museum, Etnologiska Studier 43, Göteborg.

Nielsen, A.E. 1996. Demografía y cambio social en la Quebrada de Humahuaca (Jujuy, Argentina) 700-1535 d. C. Relaciones de la Sociedad Argentina de Antropología 21:307-354.

Nielsen, A.E. 1997. Nuevas evidencias sobre la producción agrícola Inka en el sector norte de la Quebrada de Humahuaca. Estudios Sociales del NOA 1:31-57.

Nielsen, A.E. 2001. Evolución social en Quebrada de Humahuaca (AD 700-1536). En Historia Argentina Prehispánica, Tomo I, editado por E. Berberian y A.E. Nielsen, pp. 171-264. Ed. Brujas, Córdoba.

Nielsen, A.E. 2006a. Pobres jefes: aspectos corporativos en las formaciones políticas preincaicas de los andes circumpuneños. En Contra la Tiranía Tipológica en Arqueología: Una Visión desde Sudamérica, editado por C. Langebaek y C. Gnecco, pp. 120-150. Universidad de los Andes, CESO, Bogotá.

Nielsen,A.E. 2006b. Plazas para los antepasados: Descentralización y poder corporativo en las formaciones políticas preincaicas en los Andes circumpuneños. Estudios Atacameños 31:63-89.

Nielsen, A.E. 2007. El Período de Desarrollos Regionales en la Quebrada de Humahuaca: aspectos cronológicos. En Sociedades Precolombinas Surandinas: Temporalidad, Interacción y Dinámica Cultural del NOA en al Ámbito de los Andes Centro-Sur, editado por V.I. Williams, B.N. Ventura, A.B.M. Callegari y H.D. Yacobaccio, pp. 235-250. Artes Gráficas Buschi S.A., Buenos Aires.

Oliszewski, N. 2012. La variabilidad racial del maíz y los cambios sociales durante el $1^{\circ}$ y $2^{\circ}$ milenio d.C. en el Noroeste Argentino. En Las Manos en la Masa: Arqueologías y Antropologías de la
Alimentación en Sudamérica, editado por P. Babot, F. Pazzarelli y M. Marschoff, pp. 271-298. Ed. Corintios 31, Córdoba.

Olivera, D.E. 1992. Tecnología y Estrategias de Adaptación en el Formativo (Agro-Alfarero Temprano) de la Puna Meridional Argentina. Un Caso de Estudio: Antofagasta de la Sierra (Pcia. de Catamarca, R.A.). Tesis de Doctorado, Facultad de Ciencias Naturales, Universidad Nacional de La Plata, La Plata.

Olivera, D.E. y J. Grant 2009. Puestos de altura de la Puna argentina: zooarqueología de Real Grande 1 y 6 y Alero Tomayoc. Revista del Museo de Antropología 2:151-168.

Olivera, D. y J. Palma 1986. Sistemas adaptativos prehispánicos durante los períodos agroalfareros de la Quebrada de Humahuaca, Jujuy, R.A. Cuadernos del Instituto Nacional de Antropología 11:75-98.

Olivera, D.E. y H.D. Yacobaccio 1999. Estudios de paleodieta en poblaciones humanas de los Andes del sur a través de isótopos estables. Actas del V Congreso Nacional Asociación Española de Paleopatología, Alcalá la Real. https://webs.ucm. es/info/aep/congreso.htm

Ortiz, G. 2007. La Evolución del Uso del Espacio en las Tierras Bajas Jujeñas (Subárea del Río San Francisco). Tesis de Doctorado, Facultad de Filosofía y Humanidades. Universidad Nacional de Córdoba, Córdoba.

Ortiz, G. y V.A. Killian Galván 2016. El consumo como vía para comprender economías mixtas. Su aplicación al sur del valle de San Francisco, Región Pedemontana de Jujuy (Argentina). En La Montaña Tropical Sur-Central y Zonas Adyacentes Desarrollos, Políticos, Intercambio e Interacción Cultural Inter-Regional, editado por S. Alconini, pp. 263-282, Editorial Plural, La Paz.

Otero, C. 2013. Producción, Uso y Circulación de Bienes en el Pucará de Tilcara (Quebrada de Humahuaca, Jujuy). Tesis de Doctorado, Facultad de Filosofía y Letras, Universidad de Buenos Aires, Buenos Aires.

Otero, C. y M.N. Tarragó 2017. Reconstructing Inca socioeconomic organization through biography analyses of residential houses and workshops of Pucara de Tilcara (Quebrada de Humahuaca, Argentine). Journal of Anthropology and Archaeology 5 (1):55-72.

Otten, J.J., J.P. Helliwig y L.D. Meyers 2006. DRI, Dietary Reference Intakes: The Essential Guide to Nutrient Requirements. National Academies Press, Washington, DC.

Palma, J. y D.E. Olivera 1992/93. Hacia la contrastación de un modelo arqueológico para el Formativo Regional en Humahuaca: el caso de Estancia Grande. Cuadernos 14:237-260.

Panarello, H.O., C.M. García, S.A. Valencio y E. Linares 1980. Determinación de la composición isotópica del carbono en carbonatos, su utilización en hidrogeología y geología. Revista de la Asociación Geológica Argentina 35 (4):460-466.

Passey, B.H., T.F. Robinson, L.K. Ayliffe, T.E. Cerling, M. Sponheimer, M.D. Dearing, B.L. Roeder y J.R. Ehleringer 2005 Carbon isotope fractionation between diet, breath $\mathrm{CO} 2$, and bioapatite in diferent mammals. Journal Archaeological Science 32 (10):1459-1470.

Pestle, W.J., C. Torres-Rouff, M. Hubbe, F. Santana, G. Pimentel, F Gallardo y K.J. Knudson 2015. Explorando la diversidad dietética en la prehistoria del desierto de Atacama: un acercamiento a los patrones regionales. Chungara Revista de Antropología Chilena 47 (2):201-209.

Quesada, M.N. 2007. Paisajes Agrarios del Área de Antofalla. Procesos de Trabajo y Escalas Sociales de la producción agrícola 
(Primer y Segundo Milenio d. C.) Tesis de Doctorado, Facultad de Ciencias Naturales y Museo, Universidad Nacional de La Plata, Buenos Aires.

Quesada, M.N., M. Gastaldi y M.G. Granizo 2012. Construcción de periferias y producción de lo local en las cumbres de El Alto-Ancasti. Relaciones de la Sociedad Argentina de Antropología 37:435-456.

Raffino, R.A. 1993. Inka. Arqueología, Historia y Urbanismo del Altiplano Andino. Editorial Corregidor, La Plata.

Raffino, R.A. 2007. Poblaciones Indígenas en Argentina: Urbanismo y Proceso Social Precolombino. Emecé Editores, Buenos Aires.

Rivolta, M.C. 1996. Calle Lavalle y Sorpresa: aportes a la investigación arqueológica de la Quebrada de Humahuaca. En XXV Aniversario Museo Arqueológico Dr. Eduardo Casanova, pp. 129-135. Instituto Interdisciplinario Tilcara (Facultad de Filosofía y Letras, Universidad de Buenos Aires), Buenos Aires.

Rivolta M.C. 2005. Cambio Social en la Quebrada de Humahuaca en Tiempos Prehispánicos (1100 - 1400 d.C.). Instituto Interdisciplinario Tilcara, Facultad de Filosofía y Letras, Universidad de Buenos Aires, Buenos Aires.

Rodríguez, J. 2009. Descripción de los sistemas económicos productivos actuales de la Quebrada de Humahuaca. Http://www. cauqueva.org.ar.

Santana-Sagredo, F., M. Hubbe y M. Uribe 2016. Isotopic evidence for marine consumption and mobility in the Atacama Desert (Quillagua, Northern Chile). International Journal of Osteoarchaeology 26 (3):476-489.

Samec, C.T., H.D. Yacobaccio y H.O. Panarello 2018. Stable isotope compositions of South American camelids in the Dry Puna of Argentina: A frame of reference for the study of prehistoric herding and hunting strategies. Journal of Archaeological Science: Reports 18:628-636

Seldes, V. 2007. Aportes de la bioarqueología al estudio de la complejidad y la desigualdad social en la Quebrada de Humahuaca (Jujuy, Argentina), Tesis de Doctorado, Facultad de Filosofía y Letras, Universidad de Buenos Aires, Buenos Aires.

Somerville, A.D., P.S. Goldstein, S.I. Baitzel, K.L. Bruwelheide, A.C. Dahlstedt, L. Yzurdiaga y M.J. Schoeninger 2015. Diet and gender in the Tiwanaku colonies: Stable isotope analysis of human bone collagen and apatite from Moquegua, Peru. American Journal of Physical Anthropology 158 (3):408-422.

Staller, J.E. 2006. The social, symbolic and economic significance of Zea mays L in the Late Horizon period. En Histories of Maize. Multidisciplinary Approaches to the Prehistory, Linguistics, Biogeography, Domestication and Evolution of Maize, editado por J. Staller, R. Tykot y B. Benz, pp. 449-467. Academic Press, Burlington.

Tarragó, M. 2000. Chacras y pukara. Desarrollos Sociales Tardíos. En Nueva Historia Argentina, editado por M. Tarragó, pp. 257-300. Ed. Sudamericana, Buenos Aires.

Tarragó, M. y M.E. Albeck 1997. Fechados radiocarbónicos para el sector medio de la Quebrada de Humahuaca. Avances en Arqueología 3:101-129.

Tieszen, L. 1991. Natural variations in the Carbon Isotope Values of plants: implications for archaeology, ecology, and paleoecology. Journal of Archaeological Science 18:227-248.

Tomczak, P.D. 2003. Prehistoric diet and socioeconomic relationships within the Osmore Valley of southern Peru. Journal of Anthropological Archaeology 22 (3):262-278.

Tykot, R.H. 2004. Stable isotopes and diet: you are what you eat. En Proceedings-International School of Physics "Enrico Fermi", Course CLIV, editado por M. Martini, M. Milazzo y M. Piacentini, pp. 433-444. IOS Press, Amsterdam.

Vidal-Elgueta, A., E. Salazar, L.F. Hinojosa, M. Uribe y S. Flores 2016. Variabilidad fenotípica en maíz (Zea mays) del sitio de Caserones-I, Región de Tarapacá. Revista Chilena de Antropología 34:31-39.

Yacobaccio, H. D. 1997. Sociedad y ambiente en el NOA precolombino. En De Hombres y Tierras, una Historia Ambiental del Noroeste Argentino, editado por C. Reboratti, pp. 26-28. Proyecto Desarrollo Agroforestal en Comunidades Rurales del Noroeste Argentino, Salta.

Zaburlin, M.A., V. Seldes y P. Mercolli 2006. Reflexiones sobre los últimos rescates arqueológicos en Tilcara. En Problemáticas de la Arqueología Contemporánea. Publicación del XV Congreso Nacional de Arqueología Argentina, compilado por A. Austral y M. Tamagnini, pp. 325-349. Universidad Nacional de Río Cuarto, Córdoba. 